\title{
La présence du rêve dans les journaux personnels d'Henry Bauchau
}

\author{
L'homme est un mendiant quand il pense... \\ mais un géant quand il rêve \\ Hölderlin cité par Henry Bauchau \\ Passage de la Bonne-Graine
}

Au moment de la rédaction de cet article, sept volumes ${ }^{\mathbf{1}}$ du journal personnel d'Henry Bauchau ont été publiés. Ces livres portent des titres qui, outre la référence à la pratique de l'écriture quotidienne ("Journal »), renvoient soit à des circonstances de la vie de l'auteur (Les Années difficiles, Passage de la Bonne-Graine), soit à l'élaboration d'œuvres de fiction (Journal d'Antigone). Cette hétérogénéité des désignations invite à s'interroger sur leur statut générique. S'agit-il de journaux intimes ? De journaux de création ? De journaux spirituels ? Une lecture attentive permet de valider toutes ces pistes. Elle permet aussi de constater qu'un fil parcourt ces livres : celui du rêve, de la présence récurrente au sein d'un ensemble discursif hétérogène, de narrations oniriques quelquefois, mais assez rarement, associées à des éléments d'interprétation.

L'importance du rêve pour l'écriture, la quête spirituelle et le travail d'analyste d'Henry Bauchau n'est pas à démontrer. Temps du rêve est le titre du livre publié en 2012 à partir d'un manuscrit de jeunesse et, dans la préface de celui-ci, est citée une phrase de Rabindranath Tagore : "que le rêve est une réalité aussi importante que la vie ». Des récits de rêve figurent dans la quasi totalité des textes qu'il a publiés, y compris dans les romans, alors que les narrations oniriques enchâssées sont devenues moins fréquentes dans l'écriture narrative contemporaine.

Tous les rêves d'Henry Bauchau ne se trouvent pas dans son journal. Certains rêves fondamentaux comme celui du rat dans La Déchirure, n'apparaît que dans le contexte de ce livre. Il arrive aussi que le diariste fasse référence à un " journal des rêves $"^{\mathbf{2}}$, exclusivement centré sur cette pratique. Les récits de rêve figurant dans les journaux n'en constituent pas moins un vaste corpus, qui tout à la fois se prête à une lecture autonome et peut être considéré comme un ensemble de textes matriciels pour les romans et la poésie, voire les ouvres théâtrales. C'est ce double statut qui sera interrogé dans les lignes qui suivent.

1 Cette étude portera sur les sept volumes : La Grande Muraille. Journal de La Déchirure (19601965), Dialogue avec montagnes. Journal du Régiment noir (1968-1971), Les Années difficiles. Journal 1972-1983, Jour après jour (1984-1988), Journal d'Antigone (1989-1997), Passage de la Bonne-Graine. Journal (1997-2001) et Le Présent d'incertitude. Journal 2002-2005.

2 On lit par exemple : "J'ai continué à tenir régulièrement mon Journal des rêves, le Journal, lui, est resté en panne" (PI, p. 197). 
Le phénomène du rêve consiste en l'apparition nocturne d'images, plus rarement de bribes de phrases, empruntées à l'expérience diurne du rêveur. Un accord semble avoir été trouvé, y compris dans les neurosciences, autour de l'idée, jadis avancée par Freud, selon laquelle le rêve se fonde sur un processus de figuration, de mise en image de désirs et de souvenirs. Le rêve est une expérience principalement visuelle. On peut le considérer comme une " pensée en images ", qui prend momentanément le dessus sur la pensée abstraite et se fonde sur une autre temporalité : celle de l'image et pas celle du discours, même si paradoxalement c'est par le discours que le rêve est transmis.

Narrer un rêve est en effet toujours un acte paradoxal car la nature de l'expérience onirique est étrangère d'une part à la logique de l'être adulte, d'autre part au principe de l'enchaînement linéaire qui régit le discours narratif. Celui qui narre ses songes doit trouver des stratégies particulières pour dire l'absurdité des situations, les métamorphoses de lieux et les incertitudes identitaires. Pour cela, il doit déconstruire le récit canonique, notamment dans son rapport à une temporalité linéaire pour dire l'apparition d'images. En outre la manière de narrer les rêves et plus encore chez Henry Bauchau qui a un parcours d'analyste - a été fortement influencée par les récits figurant dans le livre fondateur de Freud: L'Interprétation des rêves, à l'origine d'une véritable rhétorique onirique, fondée notamment sur l'usage du présent comme temps verbal et le fait de commencer le récit par des compléments de lieux rappelant des didascalies dans un texte théâtral.

L'écriture diaristique d'Henry Bauchau n'accorde que progressivement une place aux récits de rêve. Ils sont peu nombreux dans La Grande Muraille. La notion de rêve est alors étroitement associée pour lui à la psychanalyse. On lit dans ce premier journal : "Je me rappelle le sentiment singulier que je ressentais au début de l'analyse lorsque Blanche me demandait de "produire" des rêves. J'étais choqué comme par l'intervention d'un procédé mécanique dans le monde intime. » (GM, p. 38.)

Les récits de rêve sont de plus en plus nombreux dans les deux volumes suivants et ont indéniablement un rôle de soutien dans une période de vie difficile. Dans Dialogue avec les montagnes, le diariste remarque : "Durant cette période j'ai fait de très nombreux rêves, presque chaque jour, ce qui montre un besoin de compensation à une période où le mauvais temps et la monotonie m'ont souvent paru accablants. " (DM, p. 137.) Ces deux volumes ont été écrits durant la période où Henry Bauchau vivait en Suisse, dirigeait l'école de Montesano, puis a été contraint de la fermer et de vendre ses biens. Il semble que le fait de se mettre à l'écoute des éléments inconscients qui s'expriment dans les rêves lui ait apporté un certain apaisement.

On peut penser qu'il en est de même avec le sixième volume, Passage de la Bonne-Graine, où le lâcher-prise que suppose l'écoute onirique constitue pour le diariste une aide, lors de la traversée d'une nouvelle période difficile liée au décès de son épouse. Effectivement les récits de rêves sont particulièrement nombreux dans ce livre. Bauchau semble l'avoir par la suite regretté. On lit dans le volume 
suivant : "J'ai eu mon frère au téléphone, il a lu Passage de la Bonne-Graine, qu'il a aimé. Il trouve comme Jacques Devriend qu'il y a un peu trop de rêves. »(PI, p. 47.)

La fréquence des récits de rêve devient moindre dans le dernier volume. Dans une entrée du 6 janvier 2004, le diariste se réjouit d'arriver de nouveau à se souvenir de ses rêves. Il met la difficulté éprouvée précédemment à les conserver au compte de la rédaction de L'Enfant bleu : "Comme je l'ai remarqué à d'autres occasions, le souvenir des rêves de la nuit, si souvent impossible quand je travaille à une ouvre d'importance, est redevenu fréquent depuis la fin du roman. "(PI, p. 132.) Il semble également que, dans ce dernier volume, la capacité d'écoute du diariste soit presque entièrement absorbée par les Autres (les différentes personnes qui viennent le voir ou qu'il rencontre) et la nature qu'il observe attentivement. On ne peut qu'adhérer à l'idée formulée par Isabelle Vanquaethem, dans un article consacré aux journaux d'Henry Bauchau, selon laquelle il y aurait dans ce volume une modification de l'écriture et un déplacement d'intérêt vers le monde extérieur :

Au paysage intérieur succède, sans l'évincer aucunement, le paysage extérieur comme objet privilégié de captation scripturale. Après avoir délié tant les nouds intérieurs de son histoire personnelle que ceux de l'Histoire, en regard de la psychanalyse qui l'a tant influencé, l'écrivain s'ouvre peu à peu à l'intemporel et se cherche ailleurs qu'en lui-même. ${ }^{3}$

La manière dont Henry Bauchau transcrit ses rêves est extrêmement variable. Certains n'occupent qu'une phrase, font l'objet seulement d'une mention rapide. On peut lire dans Dialogue avec les montagnes : "Au XVe siècle, des femmes fines et ouvragées, mystérieuses comme on en voit dans la peinture de ce temps. Puis sur une route un énorme insecte volant. " (DM, p. 75.) Et, dans Passage de la Bonne-Graine : "Présence de mon père. Il est question de la gloire militaire. " (PBG, p. 351.) Il s'agit de transcriptions minimales, au plus près de l'apparition nocturne. D'autres rêves sont réduits à la mention d'une couleur ou d'une sensation.

Les récits sont quelquefois annoncés par la mention "Rêve " ou "Rêve $d u$... ". D'autres rêves ne sont pas directement racontés mais apparaissent lors de passages rapportant des entretiens ; c'est notamment le cas pour un grand nombre de rêves figurant dans Dialogue avec les montagnes, où les narrations oniriques figurent à l'intérieur de comptes rendus des entretiens avec le Dr Dreyfuss.

Henry Bauchau écrit presque toujours ses rêves au présent et les introduit souvent par des phrases nominales indiquant leur cadre. On lit au début d'un récit de rêve dans Passage de la Bonne-Graine: "Une grande chambre où il y a une réunion à la fois familiale et amicale»(PBG, p. 195), et dans un autre rêve : "Grande maison, grande famille, on me montre les œuvres d'un jeune garçon de quatorze ans " (PBG, p. 399). On perçoit l'influence du type de récits figurant dans L'Interprétation des

3 Isabelle Vanquaethem, "Les journaux d'Henry Bauchau : de moi à soi, se parcourir ", dans Jean Leclercq et Nicolas Monseu, Phénoménologies littéraires de l'écriture de soi, Dijon, Éditions universitaires de Dijon, 2009, p. 221-222. 
rêves de Freud. Ailleurs, la phrase "Une scène se passe [...]" (AD, p. 190) débutant la narration du songe révèle le fait que, pour le diariste, l'expérience onirique ressemble à celle de l'intervention de personnages sur une scène de théâtre.

Henry Bauchau n'interprète que rarement ses rêves. Dans le Journal d'Antigone, il cite avec plaisir une lettre de Marie-Claire Boons rédigée à la suite de la lecture de son journal dans laquelle elle écrit : "Je suis heureuse que tu ne sois pas tombé dans le piège de l'interprétation des rêves : seul, le réseau qu'ils composent délivre pour chacun ce qu'ils disent. " (JA, p. 204.) Cette conception plutôt littéraire et poétique du rêve semble le guider. Elle ne connaît que quelques exceptions. Les rêves liés aux séances d'entretien avec le Dr Dreyfuss, nombreux dans Dialogue avec les montagnes, sont suivis d'interprétations, proposées par l'un ou l'autre des deux interlocuteurs.

C'est ainsi qu'on ne trouve que très rarement dans ces journaux la disposition canonique freudienne, consistant à faire suivre le récit du rêve de la reprise de chaque terme ou élément qui suscite la libre association. Le diariste le fait pour un rêve dans Jour après jour, où chaque élément est repris, suivi du verbe "évoque " et des associations correspondantes (JJ03, p. 235). Pour quelques rêves de Passage de la Bonne-Graine, il procède également par associations, terme à terme ${ }^{4}$. De même, dans ce livre, plusieurs rêves sont accompagnés de la mention " désir du rêve " et d'un essai de reconstitution du désir inconscient sous-jacent à l'apparition onirique.

Dans les journaux de Bauchau, les rêves sont présents pour eux-mêmes, pour ce qu'ils introduisent de déconstruction au sein de la rationalité du compte rendu quotidien et non pour ce qu'ils signifient quant à l'histoire personnelle du rêveur. Le diariste échappe à la tentation de l'autoanalyse et, si la pratique du récit de rêve l'aide à vivre et à traverser les périodes difficiles de l'existence, cette démarche ne passe pas par la conscience, ni par l'analyse et l'interprétation.

On peut également remarquer qu'il y a chez l'auteur d'Edipe sur la route, à l'instar des écrivains surréalistes, perméabilité entre le songe et la vie éveillée, présence du songe dans la vie réelle et du réel dans le songe. Le diariste évoque de nombreux moments de rêverie, diurne ou nocturne, où son activité psychique est à la fois commandée par le moi et abandonnée aux images. Dans Les Années difficiles, il est question d'un " demi-rêve éveillé ". Dans le Journal d'Antigone, il parle de "longue rêverie commencée sans doute sous la forme d'un rêve "(JA, p. 386). Il arrive également qu'il fasse référence à l'expérience - bien connue de ceux qui notent leurs songes - d'une intervention du moi vigile au sein de l'expérience du rêve.

Contrairement à l'orthodoxie freudienne et ici encore à l'instar des surréalistes, Bauchau ne met pas seulement le rêve en relation avec son passé individuel mais le considère comme une instance qui dépasse sa personne, susceptible de donner des informations sur l'avenir ou d'être un guide lors de décisions. Les termes qu'il

$4 \quad$ Voir PBG, p. 174, 204, 351 et 358. 
utilise pour parler du rêve sont révélateurs du statut qu'il donne à cette expérience. Le rêve est pour lui un message ("Nombreux messages nocturnes au cours de cette nuit entrecoupée » : GM, p. 294 ; "Un rêve me dit [...] " : JA, p. 318), une question ("Le rêve m'interroge ": AD, p. 361 ; "Ce rêve m'interroge, je me demande s'il ne conteste pas [...] » : JA, p. 473), un avertissement ("Le rêve où je voulais prendre des raccourcis dangereux dans un tournant où je manque un rendez-vous [celui de Mao] m'avertit ": AD, p. 382). On lit également : "Le rêve m'a ouvert la porte " (JJ03, p. 32), "Un rêve de cette nuit me fait voir [...] " (JJ03, p. 286). Le rêve est personnifié, contient un message de l'inconscient, semble toujours vouloir en dire plus que ce que le diariste est en mesure de saisir. Dans Passage de la Bonne-Graine, figurent les expressions " don du rêve " (PBG, p. 91) et "l'aveu du rêve " (PBG, p. 241). Le rêve peut amener le diariste à des changements d'attitude dans sa vie.

Henry Bauchau joue avec l'idée selon laquelle le rêve pourrait être prémonitoire. Dans la période où il tente de vendre les maisons qu'il possède en Suisse, il fait un rêve dans lequel il nage dans une piscine et se cogne contre la paroi ( $\mathrm{AD}, \mathrm{p} .47$ ). Il fait ce rêve le 2 octobre 1972 et le 15 janvier 1973, non seulement il remarque que cela lui est arrivé dans la réalité mais il signale qu'au moment où il a ouvert son journal, il est tombé " par hasard " sur la page du 3 octobre où il avait noté son rêve. Dans Les Années difficiles, il écrit "Le rêve semble bien prémonitoire " (AD, p. 214) à propos d'un songe fait la veille d'un séjour à l'hôpital.

Quant aux contenus des rêves (les lieux, les personnages, les événements), ils apparaissent d'une extrême richesse et diversité dans l'ensemble des récits donnés à lire dans le journal. Comme ceux de tout être humain sans doute, les récits de rêve d'Henry Bauchau se partagent entre rêves bizarres, riches en métamorphoses de lieux et d'objets et rêves liés aux proches, aux membres de la famille, permettant au sujet de revivre des moments de l'enfance et de revoir en songe des êtres disparus.

Les rêves marqués d'étrangeté sont, dans l'ensemble du corpus, moins fréquents que les rêves où apparaissent des proches (morts ou vivants). Quelques exemples de rêves où dominent la bizarrerie et les métamorphoses peuvent être trouvés dans Les Années difficiles. Dans un rêve, Henry Bauchau est au volant d'une voiture en compagnie d'Ariane Mnouchkine et cette voiture se transforme en charrette avec un petit cheval (AD, p. 197). Dans un autre, il rêve que sa compagne, Laure, a fait des dessins pour Le Canard enchaîné et les vend pour un bon prix (AD, p. 267). Également dans Les Années difficiles, on peut lire cet étrange récit : "Cornelia dans le métro. Tient une clef ou une coupe de champagne. Il y a un trou dans le vert qui signifie qu'il faut dépasser le stade de Médée. "(AD, p. 360.) Le diariste ne commente pas l'homophonie évidente verre/vert et se contente de livrer sans autre commentaire cet étrange songe au lecteur de son journal. Même impression de mystère et d'étrangeté dans ces deux rêves extraits de Jour après Jour : "J'aide à la libération d'un prisonnier. C'est d'abord un homme, il apparaît sous la forme d'un pigeon bleu qui est le Saint-Esprit. C'est aussi une femme, héroïne de la guerre de libération » 
(JJ03, p. 33), "Un jeune spadassin, en armure brillante. Il entre dans la vie politique et finalement devient pape "(JJ03, p. 235). Dans Passage de la Bonne-Graine, le diariste narre comment dans un rêve lui est apparue une étrange machine où on insérait une fille et un garçon et comment il a été amené à prendre place derrière une jeune fille nue (PBG, p. 303). Ailleurs dans le même livre, il raconte un rêve où il a dû ramper et monter à l'intérieur d'une sorte de tuyau (PBG, p. 401).

Majoritaires sont cependant les songes où apparaissent des lieux et des personnages liés à la vie diurne du rêveur. Les lieux des rêves d'Henry Bauchau sont souvent ceux de son enfance : Crimont, Archennes (et notamment l'étang d'Archennes dont la présence est récurrente dans les rêves). Mais aussi ceux de sa vie adulte : Montesano, Paris, des lieux de vacances ou qui concernent son écriture (comme la Chine au moment de la rédaction du livre sur Mao).

Ses parents apparaissent dans plusieurs de ses rêves avec un mélange d'époques. Quelquefois lui-même est enfant ; d'autres fois, non. Plusieurs rêves le ramènent en arrière vers l'enfance de ses enfants, alors que ceux-ci ont grandi. Son ami Théo Léger est très souvent présent dans les narrations oniriques, de même que Laure, notamment dans Passage de la Bonne-Graine, à l'époque de sa maladie et de son décès. Henry Bauchau rêve aussi très souvent du couple Pierre Jean Jouve et Blanche Reverchon. La présence de celle qui a été son analyste et lui a ouvert la voie de l'écoute de l'inconscient n'a rien pour surprendre. Plus étonnante est la récurrence des apparitions d'Ariane Mnouchkine, tellement fréquente que le diariste l'a mentionnée avec surprise sans pour autant pleinement la comprendre. "Je m'interroge sur la fréquence de l'apparition du Théâtre du Soleil et d'Ariane dans mes rêves ", lit-on dans Le Présent d'incertitude (PI, p. 38). Henry Bauchau rêve également des personnages de ses livres : Mao, Edipe, Antigone, Diotime. Mais aussi de personnages de la littérature, comme le Duc de Guermantes.

Lui-même l'a remarqué : de nombreux personnages célèbres contemporains ou décédés apparaissent dans ses rêves. Il s'agit d'hommes politiques (de Gaulle, etc.), de grands écrivains des générations antérieures (Mallarmé, Claudel, Gide, Sartre, Malraux, Camus). "Cette nuit, je rêve que Giscard et Debré se battent avec des sabres de bois. [...] Le rêve me dit qu'il y a en moi du Giscard, du Debré et qu'il faut combattre avec autre chose que des sabres de bois ", lit-on dans Dialogue avec les montagnes (DM, p. 18). Et, dans Les Années difficiles : "Mes rêves le révèlent cruellement, j'aurais voulu "jouer un rôle", je me suis cru des aptitudes à la vie publique. ” (AD, p. 203). Le rêve permet un retour vers l'imaginaire enfantin de toute puissance et explique que le sujet de l'expérience onirique se voit entouré de figures célèbres.

De nombreux rêves font référence à des situations de compétition. Bauchau a lui-même mis ce type de rêve en relation avec son expérience enfantine de lutte avec son frère aîné, Jean, combats dans lesquels il n'avait pas le dessus mais qui lui 
permettaient de lutter". C'est ainsi qu'on peut lire dans La Grande Muraille : "Rêve important fait sur le thème de deux adversaires dont l'un demande à l'autre de lui forger une arme pour reprendre le combat " (GM, p. 136) et dans Dialogue avec les montagnes : "Rêvé cette nuit d'un mouton qui jouait avec moi à l'agressivité [...]. Tout le rêve portait sur le rapport fort-faible. " (DM, p. 19). Dans Jour après jour, il rêve d'un combat collectif avec assaut, d'une course de voitures à laquelle il participe, de Maeterlinck se battant en duel. Dans Passage de la Bonne-Graine, il se rêve entraîneur d'une nageuse.

Henry Bauchau narre aussi de nombreux rêves où apparaissent des animaux, avec une nette prédominance des chevaux et des bêtes sauvages. Il rêve de sangliers, d'hippopotames et de lynx, de lionnes, de tigres et de loups. Le diariste sait que l'apparition d'animaux en songe a une signification importante en relation avec l'inconscient du dormeur. Il le commente, dans Dialogue avec les montagnes, à propos d'un rêve dans lequel sa sœur n'arrive pas à maîtriser un cheval et où apparaît un sanglier : " un grand sanglier sort du bois, il est d'un gris-noir, énorme, impressionnant. Je l'admire et je suis intéressé par lui comme je le suis par les grandes manifestations de l'inconscient " (DM, p. 157). Dans le même livre, un rêve est narré deux fois (directement et dans la transcription du dialogue avec le Robert Dreyfuss) : celui de l'apparition d'un très beau cheval bleu "très puissant " (DM, p. 280 et 285). Dans Les Années difficiles, c'est un " magnifique loup gris-bleu avec des reflets argents " qui lui apparaît dans son sommeil. Le diariste commente : "M'éveille, très impressionné par la beauté du loup, avec le sentiment d'un rêve important » (AD, p. 214).

Les paysages dans lesquels se déroulent ces rêves sont également empreints d'une grande force et beauté. On peut noter la fréquence de l'apparition de lacs, étangs, piscines, rivières, cascades et autres zones aquatiques. L'archétype de ces images semble être l'étang d'Archennes, qui joue un rôle essentiel dans l'imaginaire de l'auteur et ses perceptions sensibles. Dans Les Années difficiles, il rêve d'une piscine rouge : "À Archennes où je prends un bain dans une piscine, très belle à coloration rouge et orange. [...] Partout il y a une couleur rouge somptueuse, comme illuminée de l'intérieur, un rouge d'automne avec des colorations d'orange et d'or. Or rouge. " (AD, p. 161-162.) Ce rêve étrange et oxymorique communique une impression de fort bonheur. Dans un autre songe, l'étang d'Archennes a une couleur dorée. Dans Jour après jour, on lit le récit de rêve suivant : "Je nage dans l'étang d'Archennes. Je nage sous l'eau avec aisance et plaisir. Il n'y a ni algue, ni boue. " (JJ03, p. 172). Dans les rêves des journaux suivants, apparaissent : "Une grande et superbe vague " (JJ03, p. 394), " une cascade " (JA, p. 27), " une chute d'eau dans les montagnes " (JA, p. 83), " un ruisseau de fleurs lumineuses " (JA, p. 458), " une rivière tumultueuse " (PBG, p. 216), " un fleuve " (PI, p. 275). L'eau est, dans l'ensemble de ces récits, bénéfique.

5 Ce type de rêve apparaît dans JA, p. 253. 
Associé quelquefois au thème aquatique, celui de la montagne est aussi très présent. La montagne est le cadre fréquent de rêves situés dans la seconde moitié des journaux. L'auteur descend d'une montagne, se trouve sur des sommets, fait de la varappe, monte, descend, glisse.

Une particularité des récits de rêve d'Henry Bauchau est la présence de nombreuses phrases. Il y a certainement plus de paroles dans ces textes que dans la moyenne des narrations oniriques ${ }^{6}$. Il est fréquent qu'Henry Bauchau s'éveille avec le souvenir d'une unique phrase, très souvent mystérieuse, entendue en rêve. Dans Dialogue avec les montagnes, il rapporte un rêve dans lequel il dit : "Ce qui a manqué à Valéry c'est l'Égypte " (DM, p. 52). Dans Les Années difficiles, on lit : "Delacroix vaincra. Je trouve ces mots sur mes lèvres ce matin après plusieurs autres rêves oubliés. " (AD, p. 31.) Dans un autre rêve, il s'entend dire : "Ne séparez pas les jumelles pour le ski »(AD, p. 266). Dans Jour après jour, on peut lire : "Une seule phrase d'un rêve. C'était à une réunion d'écrivains en Angleterre. L'un d'eux disait en parlant d'une jeune romancière : "Quand elle parle à quelqu'un, même ses cheveux vont vers l'autre.” "(JJ03, p. 274.) Ces phrases résonnent comme des oracles et renforcent l'idée, sous-jacente à ces différents récits, selon laquelle quelque chose cherche à être dit dans le récit de rêve, que l'écrivain ne peut formuler autrement qu'avec la citation exacte des mots entendus.

Les exemples cités de récits de rêves révèlent la volonté d'Henry Bauchau de laisser à l'univers onirique son absurdité, d'ouvrir, par ces narrations son journal sur l'espace non maîtrisé, non contrôlable, qui donne naissance aux visions nocturnes. Les rêves sont là parce qu'ils introduisent dans l'écriture diaristique et fictionnelle des éléments infantiles, irrationnels, sans lesquels elle ne saurait exister comme ouvre littéraire à part entière.

Que le rêve est à l'origine de l'écriture littéraire de Bauchau semble évident. On peut voir facilement que certains thèmes de ses livres dérivent directement de visions nocturnes. Par exemple la fréquence des rêves de rivalité peut être mise en relation avec les passages d'Antigone autour d'Étéocle et Polynice. La fascination pour les chevaux et les animaux sauvages est aussi une dimension importante de son imaginaire tel qu'il s'exprime dans les ouvres de fiction. Les journaux témoignent à plusieurs reprises d'un effort de l'auteur pour se laisser guider par le rêve vers l'écriture. "C'est la considération fréquente de mon enfance par la mémoire et par le rêve qui, plus que toute autre chose, m'a permis de devenir psychanalyste. C'est de là aussi qu'est née ma possibilité de me délier par l'écriture ", écrit-il dans Jour après Jour (JJ03, p. 187). "Se délier par l'écriture ", c'est lâcher prise du côté de la pensée rationnelle et du contrôle, accepter que l'écriture soit un

6 Dans l'article "Le rêve entre imagerie et narrativité ", Christian Vandendorpe avance le chiffre de $6 \%$ de paroles sur un corpus de cent rêves publiés entre 1940 et 1999 (dans Christian Vandendorpe [dir.], Le Récit de rêve, Québec, Nota Bene, 2005, p. 47). On peut penser que les mots occupent davantage de place dans les rêves narrés par Henry Bauchau. 
don et que la qualité principale de l'écrivain soit d'être réceptif à ce qui se manifeste en lui dans l'expérience onirique. C'est indéniablement vers ce qu'il appelle la "pensée nocturne " qu'il se tourne pour trouver la force d'écrire. Il note dans le Journal d'Antigone :

Pendant la nuit je continue à penser à Antigone. De cette pensée nocturne émanent parfois des inspirations dont, le plus souvent, rien ne parvient à la pensée diurne. Rien, sinon une orientation sourde qui me tourne vers l'écriture, comme vers mon soleil intérieur. (JA, p. 311.)

Plusieurs livres d'Henry Bauchau sont nés de l'espace intermédiaire du rêve. Le rêve du rat a infléchi et dénoué le projet d'écriture de La Déchirure, roman qui par ailleurs se termine sur un rêve. Dans l'un des articles publiés dans L'Écriture à l'écoute, il établit une relation complexe entre la fin de sa seconde analyse, le début de l'écriture du Régiment noir et un rêve dans lequel il descend une montagne en luge et traverse un mur de neige ("La scène du rêve "- EE, p. 129).

Le premier projet d'Edipe sur la route, tel qu'il est évoqué dans les dernières pages des Années difficiles, était en relation avec une série de récits de rêves. On lit en effet dans une entrée du 6 mars 1983 :

\section{Notes pour Edipe sur la route:}

Je reprends un rêve fait dans la nuit du 27 au 28. Il s'agit d'une série de rêves faits par un analysant, parent de Freud, ou par lui-même. [...] Il s'agirait donc de rêves faits pour Edipe et/ou par lui. Rêves dont il modifie l'ordre car certains ne sont pas prêts à être analysés. (AD, p. 437.)

C'est enfin en rêve que lui apparaît le dénouement d'Antigone. On lit dans Journal d'Antigone : "Avant-hier je me suis éveillé au milieu de la nuit à la suite d'une série d'images qui sont sorties de ma mémoire mais dont le sens était qu'Antigone devait se terminer par sa montée sur la scène du théâtre » (JA, p. 156).

Ce qui est vrai de l'écriture romanesque l'est plus encore de l'écriture poétique. Dans Jour après jour, Bauchau évoque la naissance en lui de ce qu'il appelle un "rêve-poème ". Il s'agit d'un texte rêvé et noté durant la nuit, déjà sous forme de poème. Le diariste écrit :

En m'éveillant vers deux heures du matin, je me suis dit : il faut absolument noter ce rêve, c'est un récit, c'est un poème. [...] Lorsque j’ai pu retravailler mon texte les jours suivants, je me suis attaché surtout à garder la littérarité du rêve et à lui donner une forme, un accent proche du son initial, sobre et presque élémentaire, qu'il me semblait avoir entendu. (JJ03, p. 111.)

Henry Bauchau a le sentiment qu'une voix à l'intérieur de lui-même lui a "dicté " ce texte. Celui-ci, intitulé "Le Baikal ", figure avec un ensemble d'autres poèmes dans le recueil symboliquement intitulé "Les greniers du sommeil ". 
Enfin le très long poème "La Sourde Oreille ou le Rêve de Freud ", est fondé sur une expérience essentielle, celle d'un rêve fait à dix-neuf ans, dans lequel l'inventeur de la psychanalyse lui apparaît. Rêve fondateur à plus d'un titre : biographique mais aussi littéraire.

Quant au théâtre, il serait trop long de développer ce point ici mais la manière dont Bauchau narre ses rêves (avec des didascalies initiales) se fonde de toute évidence sur l'idée d'une similitude entre la scène du théâtre et celle du rêve, rapprochement explicité dans l'article "La scène du rêve ", au cours duquel l'auteur mentionne sa pièce Gengis Khan et la réplique à l'Empereur de Chine du personnage éponyme, qui se réclame du "droit du rêve ".

Avant-texte, texte-origine, texte intermédiaire ou déjà discours littéraire ? Il demeure difficile de définir le statut du récit de rêve pour Henry Bauchau. Ce qui est certain est que, parce qu'il est principalement constitué d'images, il permet au sujet de l'écriture d'entrer dans un autre type de pensée et de temporalité. Dans le rêve, il n'y pas de successivité au sens logique, pas de profondeur temporelle. Comme dans l'inconscient, le présent et le passé coïncident. Dans une entrée du 13 mars 1980, figurant dans Les Années difficiles, l'auteur rapproche son projet d'écriture de celui du rêve : "Ce que je cherche c'est un moyen de télescoper le temps, tout en permettant le récit. De condenser le temps et les personnages comme dans les rêves. " (AD, p. 352.) Le récit de rêve permet à Henry Bauchau d'entrer dans une pensée où les images dominent. Ce n'est d'ailleurs pas un hasard si la peinture comme thème ou autobiographème joue un rôle aussi important dans ses romans. Quand il évoque CEdipe, aveugle, en train de faire apparaître l'image de la vague ou quand il choisit comme personnage principal de Déluge, un peintre, il s'agit pour lui de désigner métaphoriquement un processus qui est aussi celui de l'écriture littéraire. Lui-même pratique la peinture et Lionel, modèle de L'Enfant bleu, devenu peintre a eu beaucoup d'importance pour lui sur le plan humain et affectif. On peut dire qu'Henry Bauchau appartient aux écrivains visionnaires plus qu'à ceux qui créent à partir d'une logique d'enchaînement linéaire. Les rêves ont donc dans son ouvre un statut mixte. Ce sont à la fois des avant-textes mais aussi des séquences littéraires autonomes dont la richesse imaginative rayonne au sein de la suite d'entrées quotidiennes des journaux personnels. Par la présence du rêve, ceux-ci acquièrent une part d'inconnu et d'irrationnel qui contribue à en faire des œuvres littéraires à part entière.

Annie Pibarot

Université Montpellier III 